\title{
ON RECURSIVE TREES WITH A UNIQUE INFINITE BRANCH
}

\author{
PETER CLOTE ${ }^{1,2}$
}

\begin{abstract}
In this paper we analyze the Turing degree of an infinite branch in a recursive tree $T \subseteq \omega^{<\omega}$ and its relation to the well-founded part of the tree. It is, of course, not surprising that the two notions are related, but it is of a certain technical interest (in terms of the coding procedure used) to establish the exact interrelation. An interpretation of our result in terms of a Cantor-Bendixson derivative operation on trees $T \subseteq \omega^{<\omega}$ is given.
\end{abstract}

It is well known that if a recursive tree $T \subseteq \omega^{<\omega}$ has a unique infinite branch $f \in \omega^{\omega}$, then $f$ is hyperarithmetic. Also (see [4]) for every recursive ordinal $\alpha$ there is a recursive tree $T \subseteq \omega^{<\omega}$ with a unique infinite branch $f \in \omega^{\omega}$ and, moreover, $0^{\alpha} \leqslant_{\mathrm{T}} f$. We refine these results by studying this relation between the height of the well-founded part of a tree (i.e., the set of nodes not lying on the infinite branch) and the Turing degree of the unique infinite branch.

1. Notation. We use standard recursion-theoretic notation (as in Rogers [5]). For the reader's convenience we recall some definitions. By $\{e\}(x)=y\left(\operatorname{resp} .\{e\}_{s}(x)=\right.$ $y$ ) we mean that the Turing machine with index $e$ yields the value $y$ on input $x$ (resp. yields the value $y$ on input $x$ and $y<s$ and the length of the calculation is bounded by $s)$. Let $\omega^{n}=\{\sigma: \operatorname{do}(\sigma)=n=\{0,1, \ldots, n-1\}$ and $\operatorname{rg}(\sigma) \subseteq \omega\}$. Let $\omega^{<\omega}=$ $\bigcup_{n<\omega} \omega^{n}$. For $\sigma \in \omega^{<\omega}$ we denote $\operatorname{do}(\sigma)$ by $\operatorname{lh}(\sigma)$, the length of the string $\sigma$; $\{e\}^{\sigma}(x)=y$ means that the Turing machine with index $e$ and oracle $\sigma$ yields the value $y<\operatorname{lh}(\sigma)$ on input $x$ after a computation of length $<\operatorname{lh}(\sigma)$, where the machine is allowed to ask questions of the form " $\sigma(i)=$ ?" for $i<\operatorname{lh}(\sigma)$. If a question " $\sigma(i)=$ ?" is asked, where $i \geqslant \operatorname{lh}(\sigma)$, then $\{e\}^{\sigma}(x)$ is undefined. For $A \subseteq \omega$ define

$$
\{e\}^{A}(x)=y \text { iff } \exists \sigma \in \omega^{<\omega}\left(\forall i<\ln (\sigma)[\sigma(i)=0 \leftrightarrow i \in A] \text { and }\{e\}^{\sigma}(x)=y\right)
$$

As usual, On designates a $\Pi_{1}^{1}$ set of notations for recursive ordinals. Let $\mathrm{On}_{1} \subseteq$ On be a $\Pi_{1}^{1}$ set of unique notations: for each recursive ordinal $\alpha$ there is a unique

\footnotetext{
Received by the editors November 15, 1983.

1980 Mathematics Subject Classification. Primary 03D25, 03D30.

Key words and phrases. Hyperarithmetic, well-founded part of a tree, Cantor-Bendixson rank.

${ }^{1}$ This material was presented at the American Mathematical Society Summer Institute on Recursion Theory in 1982, held at Cornell University, Ithaca, New York.

${ }^{2}$ This paper was written while the author was visiting the Department of Computer Science, University of Toronto, Toronto, Canada.
} 
noiation $a \in \mathrm{On}_{1}$ with $|a|=\alpha$. By $0^{\alpha}$ we mean $H_{a}$ (sometimes written $H(a)$ for typographical reasons), where $a \in \mathrm{On}_{1}$ and $|a|=\alpha$.

To avoid notational cumbersomeness, we use recursion theory directly on $\omega^{<\omega}$. A tree $T \subseteq \omega^{<\omega}$ is a set of finite sequences closed under initial segment, i.e., $s \in T$ and $t=s \uparrow \operatorname{lh}(t) \rightarrow t \in T$. We write [T] to denote the set of infinite branches of $T$, so $[T]=\left\{f \in \omega^{\omega}: \forall n f \uparrow n \in T\right\}$. For $s \in T$ let

$$
T_{s}=\{t \in T: t \uparrow \ln (s)=s\} \cup\{t \in T: s \uparrow \operatorname{lh}(t)=t\} .
$$

A tree $T \subseteq \omega^{<\omega}$ is recursive if $\langle T\rangle=\{\langle s\rangle: s \in T\}$ is recursive, where

$$
\langle s\rangle=\Pi\left\{p_{i}^{s(i)+1}: i<\operatorname{lh}(s)\right\}
$$

and $p_{i}$ is the $(i+1)$ st prime number. If $s \in T$ and $\left[T_{s}\right]=\varnothing$ then define, as usual, the height by

$$
\operatorname{ht}(s)=\sup \{\operatorname{ht}(t)+1: \operatorname{lh}(t)>\operatorname{lh}(s) \text { and } t \uparrow \operatorname{lh}(s)=s\} .
$$

If $\left[T_{s}\right] \neq \varnothing$ then put ht $(s)=\infty$.

Let $\gamma_{T}=\sup \left\{h t(s)+1: s \in T\right.$ and $\left.\left[T_{s}\right]=\varnothing\right\} ; \gamma_{T}$ is the ordinal that measures the height of the well-founded part of the tree $T$. In [2] we investigated the relation between the "leftmost" infinite branch of a recursive tree and the rank of the set of nodes to the left of that leftmost infinite branch. This paper extends those results.

2. Trees with a unique infinite branch. It is well known that if $T \subseteq \omega^{<\omega}$ is a recursive tree with a unique infinite branch, then that branch is hyperarithmetic. To study the interrelation between $\gamma_{T}$ and the Turing degree of that branch, we state an important result due to Chen [1]. For $\alpha$ a recursive ordinal let

$\mathrm{WF}(\alpha)=\{e: e$ codes a well-founded partial ordering on a subset of $\omega$ of rank $<\alpha\}$. If $\alpha=\lambda+n$, where $\lambda$ is 0 or a limit recursive ordinal and $n$ is an integer, let $r(\alpha)=\lambda+2 n$. Let $e_{\alpha}$ (resp. $a_{\alpha}$ ) denote a $\Sigma_{\alpha}^{0}$ (resp. $\Pi_{\alpha}^{0}$ ) complete set (see Rogers [5]).

THEOREM (CHEN [1]). If $\alpha$ is a recursive ordinal greater than 1 , then $\mathrm{WF}(\omega \cdot \alpha)$ is many-one equivalent to $e_{r(\alpha)} ; \operatorname{WF}(\omega) \equiv_{\mathrm{T}} 0^{2}$. For $n \geqslant 1$ and $\alpha$ a recursive ordinal greater than or equal to $1, \mathrm{WF}(\omega \alpha+n)$ is many-one equivalent to $a_{r(\alpha)+1}$.

Intuitively it requires $\alpha$ many blocks of quantifiers $\exists \forall$ to tell if the rank of a recursive tree without any infinite branches is less than $\omega \cdot \alpha$. The following proposition is also in [2].

Proposition 1. If $T \subseteq \omega^{<\omega}$ is a recursive tree with a unique infinite branch $f$ then $\gamma_{T} \leqslant \omega_{1}^{c k}$ (the least nonrecursive ordinal). If $\gamma_{T} \leqslant \omega \cdot \alpha$ then $f \leqslant_{\mathrm{T}} e_{r(\alpha)}$.

Proof. As the first part is obvious, we show $f \leqslant_{\mathrm{T}} e_{r(\alpha)}$. Given $f(0), \ldots, f(n)$ let $f(n+1)=$ least $x[\mathrm{ht}(\langle f(0), \ldots, f(n), x\rangle) \nless \omega \cdot \alpha]$.

To see that this is indeed the best possible, we begin with the well-known

THEOREM 2 (SEE [4]). For every hyperarithmetic set $A \subseteq \omega$ there is a recursive tree $T \subseteq \omega^{<\omega}$ with a unique infinite branch $f \in \omega^{\omega}$ and, moreover, $A \leqslant_{\mathrm{T}} f$. 
PrOOF. For the convenience of the reader, we give a sketch of this classical result. This proof was shown to us by A. Louveau. Let $p_{A} \in \omega^{\omega}$ be the principal function of $A$, i.e., $p_{A}(n)=(n+1)$ st element of $A$, when $A$ is listed in increasing order. For $g \in \omega^{\omega}, g \in\left\{p_{A}\right\} \leftrightarrow \forall n g(n)=p_{A}(n)$ so $\left\{p_{A}\right\}$ is $\Delta_{1}^{1}$. By the boundedness theorem, there is a recursive function $h: \omega^{\omega} \rightarrow \omega^{\omega}$ such that

$$
g \in\left\{p_{A}\right\} \leftrightarrow h(g) \in \mathrm{WO} .
$$

Let $\gamma$ be a recursive ordinal such that $g \in\left\{p_{A}\right\} \leftrightarrow h(g) \in$ WO and $|h(g)|<\gamma$. Set $Q(g, k) \leftrightarrow k$ codes an order preserving injection from $<_{h(g)}$ onto an initial segment of $\leqslant_{\gamma}$. Hence,

$$
g \in\left\{p_{A}\right\} \leftrightarrow \exists ! k Q(g, k) .
$$

As $Q(g, k)$ is of the form $\forall n \exists m R(g, k, n, m)$, where $R$ is recursive, set

$$
S(\langle g, k, j\rangle) \leftrightarrow \forall n\left[R(g, k, n, j(n)) \text { and } \forall n<j(n)_{\neg} R(g, k, n, m)\right] .
$$

$S$ is a $\Pi_{1}^{0}$ singleton and, hence, yields a tree $T \subseteq \omega^{<\omega}$ with a unique infinite branch $\langle g, k, j\rangle=f \in[T]=S$ and, moreover, $p_{A} \leqslant_{\mathrm{T}} f$.

For the sharp correlation between $\gamma_{T}$ and the Turing degree of the unique infinite branch of $T$, we proceed differently.

Proposition 3. There is a recursive tree $T \subseteq \omega^{<\omega}$ with a unique infinite branch $f$ such that $\gamma_{T}=\omega$ and $f \equiv_{\mathrm{T}} 0^{2}$.

Proof. It is well known that $\operatorname{Inf}=\left\{e: W_{e}\right.$ is infinite $\} \equiv{ }_{\mathrm{T}} 0^{2}$. Here $W_{e}$ is the domain of $\{e\} ; W_{e, s}=\{x<s: \exists y<s\{e\}(x)=y$ and the computation is bounded by $s$ steps $\}$, and for $A \subseteq \omega, W_{s}^{A}=\left\{x: \exists y\{e\}^{A}(x)=y\right\}$, and if $\sigma \in \omega^{<\omega}, W_{e, s}^{\sigma}=$ $\left\{x<s: \exists y<s\{e\}^{\sigma}(x)=y\right.$ and the computation is bounded by $s$ steps $\}$.

$$
\begin{aligned}
& f \in[T] \leftrightarrow f\left(p_{i}\right)=0 \text { and } \forall n \geqslant 1 \\
& \qquad f\left(p_{i}^{n+1}\right)=\text { least } s>f\left(p_{i}^{n}\right)\left[W_{i, s}-W_{i, f\left(p_{i}^{n}\right)} \neq \varnothing\right],
\end{aligned}
$$

OR

$$
\begin{array}{r}
f\left(p_{i}\right)=1,\left[f\left(p_{i}^{2}\right) \neq 0 \rightarrow\left(f\left(p_{i}^{2}\right)-1 \in W_{i, f\left(p_{i}^{3}\right)} \text { and } f\left(p_{i}^{2}\right)-1 \notin W_{i, f\left(p_{i}^{3}\right)-1}\right)\right] \\
\text { and } \forall n \geqslant f\left(p_{i}^{2}\right)\left[n \notin W_{i}\right] .
\end{array}
$$

In words, if $f$ "says" that $i \in \operatorname{Inf}$, then $\left\{f\left(p_{i}^{n}\right): n \geqslant 1\right\}$ witnesses this in a "minimal" way. If $f$ "says" that $i \notin \operatorname{Inf}$, then $f\left(p_{i}^{2}\right)=0 \rightarrow W_{i}=\varnothing$ and $f\left(p_{i}^{2}\right) \neq 0 \rightarrow f\left(p_{i}^{2}\right)=$ (maximum of $\left.W_{i}\right)+1$, and $f\left(p_{i}^{3}\right)$ gives the smallest length of computation.

It is easy to see that $0^{2} \leqslant_{\mathrm{T}} f$ and that for all $s \in T$, if $s \neq f \uparrow s$ (i.e., if $s$ does not lie on the infinite branch), then $\mathrm{ht}(s)<\omega$; hence, $\gamma_{T} \leqslant \omega$. From the above definition it is clear how to write down the definition of $T$ so that $[T]=\{f\}$. Since $\left\{i: W_{i}\right.$ has exactly $k$ elements $\}$ is infinite for each $k<\omega$, one sees that $\gamma_{T}=\omega$. By Proposition $1, f \equiv{ }_{\mathrm{T}} 0^{2}$. 
Lemma 4. Suppose $\alpha$ is a recursive successor ordinal, $U \subseteq \omega^{<\omega}$ is a recursive tree with a unique infinite branch $f_{U}$, and, furthermore, $\gamma_{U}=\omega \cdot \alpha$ and $e_{r(\alpha)} \equiv_{\mathrm{T}} f_{U}$. Then there is a recursive tree $T \subseteq \omega^{<\omega}$ with a unique infinite branch $f_{T}$ and, furthermore, $\gamma_{T}=\omega(\alpha+1)$ and $e_{r(\alpha+1)} \equiv_{\mathrm{T}} f_{T}$.

Proof. The idea is to encode $\operatorname{Inf}\left(e_{r(\alpha)}\right)=\left\{e: W_{e}^{e_{r(\alpha)}}\right.$ is infinite $\}$ into $f_{T}$ by imitating the proof of Proposition 3, but where we let a Turing machine compute "on" the given tree $U$. As $\operatorname{Inf}\left(e_{r(\alpha)}\right) \equiv_{\mathrm{T}} e_{r(\alpha+1)}$, we have $e_{r(\alpha+1)} \leqslant{ }_{\mathrm{T}} f_{T}$. By the condition on $\gamma_{T}$ and Proposition 1, we have $f_{T} \leqslant_{\mathrm{T}} e_{r(\alpha+1)}$ and, thus, $e_{r(\alpha+1)} \equiv_{\mathrm{T}} f_{T}$.

We first describe recursive trees $T^{e} \subseteq \omega^{<\omega}$ with a unique infinite branch $f_{e}$ such that the question " $e \in \operatorname{Inf}\left(e_{r(\alpha)}\right)$ " is recursive (uniformly) in $f_{e}$. First, we give some notation. As we are using recursion theory directly on $\omega^{<\omega}$, let $k: \omega \rightarrow \omega^{<\omega}$ be a recursive bijection. If $U \subseteq \omega^{<\omega}$ is a recursive tree and $s, t \in \omega$, then we write $s \prec_{U} t$ if $s \neq t, k(s), k(t) \in U$, and $k(s)=k(t)\left\lceil\operatorname{lh} k(s)\right.$. We can assume that $s \prec_{U} t \rightarrow s$ $<t$ and $k(0)=\langle\rangle$. Now define

$f \dot{\epsilon}\left[T^{e}\right] \leftrightarrow$

EITHER $\quad f(0)=0 " f$ says that $e \in \operatorname{Inf}\left(e_{r(\alpha)}\right)$ "

and $\forall n\left[f(n) \prec_{U} f(n+1)\right.$ "branch requirement"

(recall that $k(0)=\langle\rangle$ )

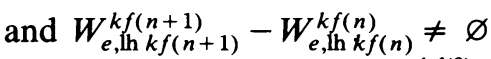

(recall $W_{e, \mathrm{~h} k f(0)}^{k f(0)}=\varnothing$ ) “requirement to

witness that $e \in \operatorname{Inf}\left(e_{r(\alpha)}\right)$ "

and $W_{e, \mathrm{hh} k f(n)}^{k f(n+1)}=W_{e, \mathrm{~h} k f(n)}^{k f(n)}$ "compatibility requirement to preserve the computation"

and $\forall s\left(f(n) \prec_{U} s{ }_{U} f(n+1) \rightarrow W_{e, \mathrm{hh} k(s)}^{k(s)}-W_{e, \mathrm{hh} k f(n)}^{k f(n)}=\varnothing\right.$ or $\left.W_{e, \mathrm{hh} k f(n)}^{k(s)} \neq W_{e, \mathrm{hh} k f(n)}^{k f(n)}\right)$

"requirement of minimality of comportment"]

OR

$f(0)=1$ " $f$ says that $e \notin \operatorname{Inf}\left(e_{r(\alpha)}\right) "$

and $\forall n \geqslant 2\left[f(n) \prec{ }_{U} f(n+1)\right.$ "branch requirement"

and $W_{e, \mathrm{~h} k f(n)}^{k f(n+1)}=W_{e, \mathrm{~h} k f(n)}^{k f(n)}$ "compatibility requirement to

preserve computation"

and $\left(f(1) \neq 0 \rightarrow f(1)-1 \in W_{e, \text { h h } k f(n)}^{k(n)}\right)$

and $\forall x<\operatorname{lh} k f(n)\left(x \geqslant f(1) \rightarrow x \notin W_{e, \mathrm{lh} k f(n)}^{k f(n)}\right)$

"condition to ensure that

$$
\begin{aligned}
& f(1) \neq 0 \rightarrow f(1)-1=\max \text { of } W_{e}^{e_{r(\alpha)}} \text { and } \\
& f(1)=0 \rightarrow W_{e}^{e_{r(\alpha)}}=\varnothing " \text { and } \ldots \\
& \forall s\left(f(n) \prec{ }_{U} s \prec{ }_{U} f(n+1) \rightarrow W_{e, \mathrm{hh} k f(n)}^{k(s)^{\prime}} \neq W_{e, \mathrm{~h} k f(n)}^{k f(n)}\right. \\
& \text { or }\left(f(1) \neq 0 \text { and } f(1)-1 \notin W_{e, \mathrm{hh} k(s)}^{k(s)}\right) \\
& \text { or } \left.\left.\exists x<\operatorname{lh} k f(n)\left[x \geqslant f(1) \text { and } x \in W_{e, \mathrm{lh} k(s)}^{k(s)}\right]\right)\right] \\
& \text { "minimality of comportment requirement". }
\end{aligned}
$$

Define $T^{e}=\left\{\sigma \in \omega^{<\omega}: \sigma\right.$ satisfies the above (replacing $f$ by $\sigma$ ) for all $\left.n<\operatorname{lh}(\sigma)\right\}$. By the "minimality of comportment" and "branch" requirements, $T^{e}$ has a unique infinite branch. Suppose $\left\langle a_{0}, a_{1}, \ldots, a_{n}\right\rangle \in T^{e}$ is a node lying on the infinite branch of $T^{e}$. 
Case 1. $a_{0}=0$.

Subcase $1 . \exists i \leqslant n k\left(a_{i}\right)$ does not lie on the infinite branch of $U$. Then let $i_{0} \leqslant n$ be the largest such $i$, so ht $T^{e}\left(\left\langle a_{0}, \ldots, a_{n}\right\rangle\right) \leqslant$ ht $_{U}\left(k\left(a_{i_{0}}\right)\right)<\omega \cdot \alpha$.

Subcase 2. $\forall i \leqslant n k\left(a_{i}\right)$ lies on the infinite branch of $U$. As ht $T^{e}\left(\left\langle a_{0}, \ldots, a_{n}\right\rangle\right) \neq \infty$, it follows that $e \notin \operatorname{Inf}\left(e_{r(\alpha)}\right)$ and a computation "on" the infinite branch of $U$ must indicate this, i.e.,

$$
\exists m\left[\forall \sigma \in T^{e}\left(\forall i<\operatorname{lh}(\sigma) \mathrm{ht}_{U} k \sigma(i)=\infty \rightarrow \operatorname{lh}(\sigma)<n\right)\right] .
$$

Hence, $\operatorname{ht}\left(\left\langle a_{0}, \ldots, a_{n}\right\rangle\right) \leqslant \omega \cdot \alpha+n<\omega \cdot(\alpha+1)$.

Case 2. $a_{0}=1$. A similar treatment yields ht $\left(\left\langle a_{0}, \ldots, a_{n}\right\rangle\right)<\omega \cdot(\alpha+1)$.

Now we sandwich together the trees $T^{e}$ by the $E$-operator (due to Chen [1]) - the $E$-operator is similar to the zig-zag enumeration of $\omega^{2}$, but applied to trees. Let $\mathrm{P}$ denote the set of codes of recursive partial orderings and WF denote the set of codes of recursive well-founded partial orderings. The rank of a well-founded partial ordering is defined as usual. The recursive ordering with code $e$ is denoted $\leqslant_{e}$.

LEMMA (CHEN [1]). Suppose $f$ is a recursive function and $f^{\prime \prime} \omega \subseteq \mathrm{P}$. Then there is a recursive partial ordering $\leqslant_{E(f)}$ such that $\leqslant_{E(f)}$ is well founded iff for some $n, \leqslant_{f(n)}$ is well founded and, in that case, $\operatorname{rank}\left(\leqslant_{E(f)}\right) \leqslant \operatorname{rank}\left(\leqslant_{f(n)}\right)+n$ for $n$ such that $f(n) \in \mathrm{WF}$.

Proof. Define

$$
\begin{gathered}
\left\langle x_{0}, \ldots, x_{m}\right\rangle \leqslant{ }_{E(f)}\left\langle y_{0}, \ldots, y_{n}\right\rangle \quad \text { iff }\left(m>n \& \forall i \leqslant n\left(x_{i}<_{f(i)} y_{i}\right) \& \forall i\right. \\
\left.\leqslant n\left(y_{i} \in \operatorname{Field}\left(\leqslant_{f(i)}\right) \& \forall j \leqslant m\left(x_{j} \in \operatorname{Field}\left(\leqslant_{f(j)}\right)\right)\right)\right) \\
\quad \text { or }\left\langle x_{0}, \ldots, x_{m}\right\rangle=\left\langle y_{0}, \ldots, y_{n}\right\rangle .
\end{gathered}
$$

Then make the calculations for the order types.

REMARK. Suppose $f$ is a recursive total function and $F^{\prime \prime} \omega \subseteq \mathrm{P}-\mathrm{WF}$. If $\{b$ : $\left.b \leqslant E_{E(f)}\left\langle a_{0}, \ldots, a_{m}\right\rangle\right\}$ is well founded, there is an $i \leqslant m$ such that $\left\{b: b \leqslant_{f(i)} a_{i}\right\}$ is well founded and $\operatorname{rank}\left(\left\langle a_{0}, \ldots, a_{m}\right\rangle\right)$ in $\leqslant_{E(f)} \leqslant \operatorname{rank}\left(a_{i}\right)$ in $\leqslant_{f(i)}$.

Proof of REMARK. The first assertion is obvious. The second assertion is proved by induction on rank $b$ for $b \leqslant_{E(f)}\left\langle a_{0}, \ldots, a_{m}\right\rangle$.

Notice that if we apply the $E$-operator to a recursive sequence of recursive trees, each having a unique infinite branch, then the resultant tree has infinitely many infinite branches. For instance, suppose $f_{i} \in\left[T_{i}\right]$ and $T$ is obtained by applying the $E$-operator to $\left\langle T_{i}: i \in \omega\right\rangle$. Then $g_{i} \in[T]$, where

$$
g_{i}(n)=\left\langle\bar{f}_{0}(i+n), \bar{f}_{1}(i+n-1), \ldots, \bar{f}_{n}(i)\right\rangle
$$

(recall that $\left.\bar{f}_{j}(k)=\left\langle f_{j}(0), \ldots, f_{j}(k)\right\rangle\right)$. This is easily alleviated by defining

$$
\begin{aligned}
& E^{*}\left(\left\langle T_{i}: i<\omega\right\rangle\right)=\{\langle\rangle\} \cup\{\sigma \in E\left(\left\langle T_{i}: i<\omega\right\rangle\right): \\
&\left.\forall i<\ln (\sigma)\left[\ln \left((\sigma)_{i}\right)=\ln (\sigma)-1-i\right]\right\} .
\end{aligned}
$$

Then it follows that $T=E^{*}\left(\left\langle T_{i}: i<\omega\right\rangle\right)$ has a unique infinite branch given by $g \in[T]$, where $g(0)=\langle\rangle$ and $g(n+1)=\left\langle\bar{f}_{0}(n-1), \bar{f}_{1}(n-2), \ldots, \bar{f}_{n-1}(0)\right\rangle$.

Now let $T=\leqslant_{E^{*}(g)}$, where $g$ is a recursive function (given by the uniformity of the $T^{e}$ 's) such that $g(e)$ is an index of $T^{e}$. 
Claim. $T$ has a unique infinite branch, $\gamma_{T}=\omega(\alpha+1)$, and $f_{T} \equiv_{\mathrm{T}} e_{r(\alpha+1)}$, where $f_{T}$ denotes the unique infinite branch of $T$.

Proof of Claim. Applying the $E^{*}$-operator to the trees $T^{e}$, each of which has a unique infinite branch, produces a tree $T$ with a unique infinite branch $f_{T}$. If $\left\langle a_{0}, \ldots, a_{n}\right\rangle \in T$ is a node not lying on the infinite branch $f_{T}$, then the above remark yields ht $\left(\left\langle a_{0}, \ldots, a_{n}\right\rangle\right)<\omega(\alpha+1)$. Hence $\gamma_{T} \leqslant \omega(\alpha+1)$. If $\gamma_{T}<\omega(\alpha+1)$ $=\omega \alpha+\omega$, then by Proposition 1 and Chen's result, we would have $f_{T} \leqslant_{T} a_{r(\alpha)+1}$, contradicting the fact that $e_{r(\alpha+1)} \leqslant{ }_{T} f_{T}$. Hence, $\gamma_{T}=\omega(\alpha+1)$. By Proposition 1, we have $f_{T} \leqslant_{\mathrm{T}} e_{r(\alpha+1)}$, so $f_{T} \equiv_{\mathrm{T}} e_{r(\alpha+1)}$.

LEMMA 5. Suppose $a=3 \cdot 5^{z} \in \mathrm{On}$ is a limit notation and uniformly in $n$ there are recursive trees $U^{n} \subseteq \omega^{<\omega}$ with a unique infinite branch $f_{n} \in\left[U^{n}\right]$ and, moreover, $\gamma_{U^{n}}=\omega|\{z\}(n)|$ and $f_{n} \equiv_{\mathrm{T}} H(\{z\}(n))$ uniformly. Then there is a recursive tree $T \subseteq$ $\omega^{<\omega}$ with a unique infinite branch $f_{T}$ and, moreover, $\gamma_{T}=\omega|a|$ and $f_{T} \equiv_{\mathrm{T}} H\left(2^{a}\right)$.

Proof. First we use the $E^{*}$-operator. Let $U=\leqslant_{E^{*}(g)}$, where $g(n)=$ an index of $U^{n}$. It is easy to check that $U$ is a recursive tree with a unique infinite branch $f_{U}$, and $\gamma_{U}=\omega|a|$, and $f_{U} \equiv_{\mathrm{T}} H(a)$. Note that if $\left\langle a_{0}, \ldots, a_{n}\right\rangle \in U$ and $\left[U_{\left\langle a_{0}, \ldots, a_{n}\right\rangle}\right]=\varnothing$, then

$$
\exists i \leqslant n\left(\left[U_{a_{i}}^{i}\right]=\varnothing \text { and } \mathrm{ht}_{U}\left(\left\langle a_{0}, \ldots, a_{n}\right\rangle\right) \leqslant \mathrm{ht}_{U^{i}}\left(a_{i}\right)\right) .
$$

This implies that if $\left[U_{\left\langle a_{0}, \ldots, a_{n}\right\rangle}\right]=\varnothing$ then ht ${ }_{U}\left(\left\langle a_{0}, \ldots, a_{n}\right\rangle\right) \leqslant \omega|\{z\}(n)|$. Using this property of $U$, we now construct a recursive tree $T$ satisfying the statement of this lemma.

Let $c_{0}$ be a fixed index such that $\forall X \subseteq \omega\left[\left\{c_{0}\right\}^{X}\right.$ enumerates the jump $X^{\prime}$ of $\left.X\right]$, where $\left\{c_{0}\right\}^{X}$ is injective. We define uniformly in $e$ the recursive tree $T^{e}$ with a unique infinite branch $f_{e}$ such that the question of " $e \in H\left(2^{a}\right)$ " is recursive in $f_{e}$. (Recall that $H\left(2^{a}\right)$ is the jump $H(a)^{\prime}$ of $H(a)$.)

Let $S^{e}=\left\{\sigma \in \omega^{<\omega}:\{e\}^{\sigma}(e)\right.$ is undefined $\}$. $S^{e}$ is clearly a recursive tree. Let $f \in\left[T^{e}\right] \leftrightarrow$

EITHER $f(0)=0$ " $f$ says that $e \in H\left(2^{a}\right)$ "

and $\forall n \geqslant 1\left(\langle f(1), \ldots, f(n)\rangle \in S^{e} \cap U\right)$ "branch requirement"

OR $f(0)=n+1$ and $\left\{c_{0}\right\}^{k f(1)}(n)=e$ " $f$ asserts that $\left\{c_{0}\right\}^{H(a)}(n)=e$ " and $\forall n \geqslant 1\left(f(n) \prec_{U} f(n+1)\right)$ "branch requirement" and $\forall s\left(s \prec_{U} f(1) \rightarrow\left\{c_{0}\right\}^{k(s)}(n) \neq e\right)$ and $\forall s \forall n \geqslant 1\left(\right.$ not $\left.f(n) \prec_{U} s \prec_{U} f(n+1)\right)$ the latter two

statements ensure "minimality of comportment".

Note that by the branch requirement in the "OR" clause, the computation is preserved, i.e., $\left\{c_{0}\right\}^{k f(n)}(n)=\left\{c_{0}\right\}^{k f(1)}(n)$.

Claim. For each $e, T^{e}$ is a recursive tree with a unique infinite branch $f_{e}$, and $\gamma_{T^{e}} \leqslant \omega|a|$ and $f_{e}(0)=0$ iff $e \in H\left(2^{a}\right)$.

PROOF OF Claim. By the branch requirement the calculations are eventually correct, and by the injectivity of $\left\{c_{0}\right\}^{H(a)}, T^{e}$ has a unique infinite branch $f_{e}$. An argument similar to that at the end of Lemma 4, using the fact that, for each $i \in \omega$, $T_{\langle i\rangle}^{e}$ is isomorphic to a subtree of $U$, yields $\gamma_{T^{e}} \leqslant \omega|a|$. 
Now let $T=\leqslant_{E^{*}(h)}$, where $h$ is a recursive function such that $h(e)=$ an index for $T^{e}$ for each $e$. Then $T$ has a unique infinite branch $f_{T}$ and $H\left(2^{a}\right) \leqslant{ }_{\mathrm{T}} f_{T}$. A computation using the earlier-mentioned property of $U$ shows that $\gamma_{T} \leqslant \omega|a|$. If $\gamma_{T}<\omega|a|$, then an application of Proposition 1 and Chen's result would yield $f_{T}<_{\mathrm{T}} H\left(2^{a}\right)$, a contradiction. Hence $\gamma_{T}=\omega|a|$ and $f_{T} \equiv_{\mathrm{T}} H\left(2^{a}\right)$.

Putting together Proposition 3 and Lemmas 4 and 5 yields

THEOREM 6. There is a recursive total function $g$ such that, for $a \in$ On $-\{1\}, g(a)$ is an index for the characteristic function of a recursive tree $T_{a} \subseteq \omega^{<\omega}$ with a unique infinite branch $f_{a}$ and, furthermore, $\gamma_{T_{a}}=\omega|a|$ and $f_{a} \equiv_{\mathrm{T}} e_{r(|a|)}$.

Proof. Let $e_{0}$ be a fixed index for the characteristic function of the tree in Proposition 3, i.e.,

$$
\left\{e_{0}\right\}(s)= \begin{cases}0 & \text { if } s \in \omega^{<\omega} \text { and } s \in T \\ 1 & \text { otherwise. }\end{cases}
$$

Let $h_{1}$ be a recursive total function such that if $a=2^{b} \in \mathrm{On}-\{1\}$ is a successor notation and if $e$ is an index of a recursive tree $U \subseteq \omega^{<\omega}$ satisfying the hypotheses of Lemma 4 , then $h_{1}(a, e)$ is the index of a tree $T$ satisfying the conclusion of Lemma 4. Let $h_{2}$ be a recursive total function such that if $a=3 \cdot 5^{z} \in \mathrm{On}-\{1\}$ is a limit notation and if $u$ is such that for all $n \in \omega,\{u\}(n)$ is an index of a tree $U^{n}$ satisfying the hypotheses of Lemma 5 , then $h_{2}(a, u)$ is an index of the tree $T$ given by the conclusion of Lemma 5 . Let $h_{3}$ be a recursive total function such that $\left\{h_{3}(k, z)\right\}(n)=\{k\}(\{z\}(n))$. The functions $h_{1}, h_{2}, h_{3}$ are obtained by the $S-m-n$ theorem using the uniformity of Lemmas 4 and 5.

Now define a recursive partial function $\psi$ by

$$
\psi(k, a)= \begin{cases}e_{0} & \text { if } a=2, \\ h_{1}(a,\{k\}(b)) & \text { if } a=2^{b}, \\ h_{2}\left(a, h_{3}(k, z)\right) & \text { if } a=3 \cdot 5^{z} \\ 0 & \text { otherwise. }\end{cases}
$$

By $S-m-n$ and the fixed point theorem, there is a $k_{0}$ such that $\left\{k_{0}\right\}(a)=\psi\left(k_{0}, a\right)$. By induction one sees that $\left\{k_{0}\right\}=g$ is a total function and satisfies the conclusion of the theorem.

Remarks. (1) Consider the Kleene-Brouwer linearization of the tree $T_{a}$. (Recall that, for $s, t \in T_{a}, s \leqslant_{\mathrm{KB}} t \leftrightarrow t=s \uparrow \operatorname{lh}(t)$ or $\exists i<\operatorname{lh}(s)[s \uparrow i=t \uparrow i$ and $s(i)<$ $t(i)]$.) This produces a recursive total ordering of order type $\beta+\delta$, where $\beta \leqslant \omega^{\omega|a|}$. It is easy to see that for the "bottom part" $B_{a}$ of the Kleene-Brouwer linearization $R_{a}$ of $T_{a}$,

we have $B_{a} \geqslant_{\mathrm{T}} e_{r(|a|)}$.

$$
B_{a}=\left\{s: \text { the set of } R_{a} \text {-predecessors of } s \text { is well-ordered }\right\},
$$

On the other hand, there is a recursive total ordering $S$ of order type $\beta+\delta$ such that for the "bottom part" $B$ of $S$,

$$
B=\{s: \text { the set of } S \text {-predecessors of } s \text { is well-ordered }\},
$$

$B$ is recursive. Hence if $f: \operatorname{Field}(S) \rightarrow \operatorname{Field}\left(R_{a}\right)$ is an order-preserving map, we have $f \geqslant_{\mathrm{T}} e_{r(|a|)}$. This observation is an essential point in the proof by H. Friedman 
that the principal axiom of $\mathrm{ATR}_{0}$ is equivalent to the statement that there is a comparison map between any two ordinals (see [3]).

(2) For a tree $T \subseteq \omega^{<\omega}$ define the derivative $D(T)=\{s \in T: h t(s) \geqslant \omega\} . D(T)$ is recursive in the double jump of $T$. Let $T^{0}=T$ and $T^{\alpha+1}=D\left(T^{\alpha}\right)$ and $T^{\lambda}=$ $\bigcap\left\{T^{\alpha}: \alpha<\lambda\right\}$ if $\lambda$ is a limit ordinal. Let $\xi_{T}=$ least $\alpha\left[T^{\alpha+1}=T^{\alpha}\right]$. Note that $\xi_{T}$ is (a modified version of) the Cantor-Bendixson rank of the tree $T \subseteq \omega^{<\omega}$. If $T \subseteq \omega^{<\omega}$ is a recursive tree then $\xi_{T} \leqslant \omega_{1}^{c k}$. If $\xi_{T}=\alpha<\omega_{1}^{c k}$, then there is an infinite branch $f \in[T]$ with $f \leqslant_{\mathrm{T}} e_{r(\alpha)}$. On the other hand, Theorem 6 yields, for each $1 \leqslant \alpha<\omega_{1}^{c k}$, an example of a recursive tree $T \subseteq \omega^{<\omega}$ with a unique infinite branch $f_{T}$ such that $f_{T} \equiv_{\mathrm{T}} e_{r(\alpha)}$ and $\xi_{T}=\alpha$. Hence, our main result yields a correlation between (a modified version of) the Cantor-Bendixson rank of a tree $T \subseteq \omega^{<\omega}$ and the Turing complexity of infinite branches of the tree: for trees with a unique infinite branch, the only possible means to obtain the branch is to successively peel off derivatives. (Note that the usual definition of the Cantor-Bendixson derivative of a tree $T$ is $D(T)=\{s \in T: \operatorname{ht}(s) \geqslant 1\}$. Consideration of Proposition 3 reveals that our definition of derivative is more adapted to calculating sharp bounds on the Turing degree of infinite branches.)

(3) R. I. Soare (oral communication) has obtained similar results for recursive trees $T \subseteq 2^{<\omega}$ of finite Cantor-Bendixson rank. In fact, recently, P. Clote, R. I. Soare, S. Wainer, and, independently, D. Cenzer and R. Smith have completed a recursion theoretic analysis of the Cantor-Bendixson theorem, showing that if $T \subseteq 2^{<\omega}$ is a recursive tree and $[T]$ is countable, then $\operatorname{rk}(T)=\lambda+n$ implies that all infinite branches of $T$ are recursive in $0^{\lambda+n}$, and that this is the best possible. Here, $\operatorname{rk}(T)$ is the usual Cantor-Bendixson rank of $T$, and $\lambda$ is either 0 or a limit recursive ordinal. These and other results will appear in a forthcoming joint paper by these five authors.

ACKNOWLEDGEMENTS. I would like to express here my thanks to K. McAloon and R. I. Soare for facilitating my attendance at the AMS Summer Institute on Recursion Theory (1982), during which meeting these results were presented. Many thanks to S. A. Cook and to the Natural Sciences and Engineering Council of Canada. The final version of this paper was typed by Mrs. Teresa Miao, whom I would like to sincerely thank, while I was visiting the Department of Computer Science at the University of Toronto.

\section{REFERENCES}

1. K. H. Chen, Recursive well-founded orderings, Ann. of Math. Logic 13 (1978), 117-147.

2. P. Clote, On the leftmost infinite branch of a recursive tree, Proc. Logic Colloq. (Jedwicin, Poland), 1981.

3. H. Friedman, Systems of second order arithmetic with restricted induction, J. Symbolic Logic 41 (1976), 557-559 (abstracts).

4. C. G. Jockusch, Jr. and T. G. McLaughlin, Countable retracing functions and $\pi_{2}^{0}$ predicates, Pacific J. Math. 30 (1969), 67-93.

5. H. Rogers, Jr., Theory of recursive functions and effective computability, McGraw-Hill, New York, 1967.

U.E.R. de Mathematiques, Universite Paris VII, 75251 Paris Cédex 05, France

Current address: Department of Computer Science, Boston College, Chestnut Hill, Massachusetts 02167 